\title{
Long-term complications of COVID-19; an updated mini-review to the current knowledge
}

\author{
Fatemeh Shafieyeh $^{*}{ }^{(}{ }^{\text {, Mahsa Heydari }}{ }^{\circledR}$ \\ Nickan Research Institute, Isfahan, Iran
}

\section{Correspondence to:}

Fatemeh Shafieyeh;

Email:elhammajlesi4@gmail.com

Received: 10 Feb. 2021

Accepted: 17 June 2021

ePublished: 20 Aug. 2021

Keywords: COVID-19, PostCOVID Syndrome, Extreme fatigue, Pulmonary injury, Cardiac injury

\begin{abstract}
In December 2019, China announced the discovery of a new coronavirus for the first time. This event has since affected the lives of people all over the world. Numerous studies have been conducted to identify the short and long-term complications of this disease. Considering the novelty of COVID-19, scientists do not have adequate information about the long-term complications of this disease; however, these longterm effects are very similar to those of other coronaviruses. researchers have recommended monitoring of COVID-19 recovered patients for identification of the long-term complications of the disease. Longterm symptoms are referred to as "Iong COVID/post-COVID syndrome", and people with these symptoms are called the "long haulers". This article gives a brief review of long-term complications of covid-19 like extreme fatigue, pulmonary fibrosis, cardiovascular complications, sensory and behavioral disorders, smell and taste dysfunctions.
\end{abstract}

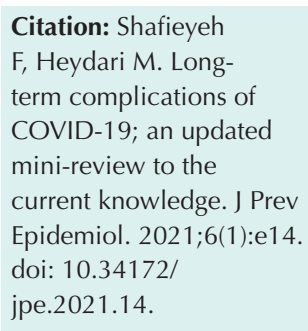

jpe.2021.14.

\section{Introduction}

In December 2019, China announced the discovery of a new coronavirus for the first time. This event has since affected the lives of people all over the world.

Experts have recommended the use of masks and disinfectants as well as social distancing to prevent the transmission of COVID-19. These measures have changed people's lifestyles.

Fever, dry coughs, shortness of breath, sore throat, headache, fatigue, and loss of sense of smell are some common symptoms of this disease (1).

Most COVID-19 patients experience mild symptoms and usually recover from the disease in 2-6 weeks; however, about 10$15 \%$ of patients display severe and critical symptoms, and in some cases, the symptoms linger for weeks or even months (2).

Old people, people with underlying diseases (e.g. hypertension, diabetes, obesity, and mental health problems), black and Asian people, and ethnic minorities are at higher risk of displaying long-term symptoms (2). However, children, adolescents, people with no history of underlying diseases, and people who have experienced mild symptoms of COVID-19 may also experience these symptoms for a long period of time (3).

Many studies have been conducted to identify the short and long-term

\section{Key point}

Long-term symptoms are referred to as "long COVID/ post-COVID syndrome", and people with these symptoms are called the "long haulers".

complications of this disease. The most common short-term complications of COVID-19 include acute respiratory distress syndrome (ARDS), acute kidney injury (AKI), proinflammatory and hypercoagulable state, thromboembolic events, cardiac injury, and sepsis (4).

Considering the novelty of COVID-19, scientists do not have adequate information about the long-term complications of this disease; however, these long-term effects are very similar to those of other coronaviruses such as the severe acute respiratory syndrome coronavirus (SARS-CoV) and the Middle East respiratory syndrome (MERSCoV) (5). Regarding the key differences between these diseases, researchers have recommended monitoring of COVID-19 recovered patients for identification of the long-term complications of the disease (6).

\section{Methods}

For this review, we searched the following international databases: Scopus, Google Scholar, Web of Science, EBSCO and PubMed for finding English language articles related

Copyright (C) 2021 The Author(s); Published by Society of Diabetic Nephropathy Prevention. This is an open-access article distributed under the terms of the Creative Commons Attribution License (http://creativecommons.org/licenses/by/4.0), which permits unrestricted use, distribution, and reproduction in any medium, provided the original work is properly cited. 
to "long-term complications of COVID-19". The keywords included COVID-19, post-COVID syndrome, extreme fatigue, pulmonary injury and cardiac injury.

\section{Long-term complaints of COVID-19}

Long-term complaints of COVID-19 recovered patients include extreme fatigue, muscle weakness, mild fever, inability to concentrate, memory loss, mood swings, sleep problems, headache, pins and needles sensations felt in the arms and legs, diarrhea and vomiting, loss of senses of taste and smell, sore throat and difficulty swallowing, onset of new diabetes and hypertension, skin rash, shortness of breath, chest or abdominal pains, coughs, palpitations and joint pains $(2,3,6,7)$.

These long-term symptoms are referred to as "long COVID/post-COVID syndrome", and people with these symptoms are called the "long haulers" $(6,8)$.

The coronavirus affects the following parts of human body:

\section{Pulmonary injury}

A mixture of ground glass opacity and consolidation are observed in lung CT scans of COVID-19 patients. These symptoms lead to prolonged pulmonary complications in $30 \%$ of the patients $(9,10)$.

COVID-19 can cause different degrees of chronic airway inflammation and pulmonary fibrosis. It can also induce lung cancer in several ways:

1. Various inflammatory factors are produced during chronic inflammation, which result in continuous stimulation of the alveolar epithelium. These factors also cause atypical hyperplasia, metaplasia, and even cancer during repair and proliferation.

2. The development of COVID-19 can lead to the release of inflammatory mediators (e.g. proteases and oxygen free radicals) through the accumulation of inflammatory cells (e.g. neutrophils and macrophages) in the alveoli. These mediators can in turn lead to fibroblast migration, proliferation, resistance to apoptosis, metaplasia and eventually cancer.

3. In addition, the large number of apoptotic cells observed in the spleen, lungs, lymph nodes, lung cells, lymphocytes, and monocytes can lead to the suppression of the immune system and thereby result in lung cancer (11).

The American Thoracic Society recommends the use of chest X-ray 12 weeks after recovery, as the resolution of imaging evidence is improved and irreversible changes are detected during this time. However, the use of chest X-ray is not recommended for examination of fully recovered patients (9).

\section{Cardiac injury}

Cardiac imaging results show inflammation of the heart muscle (i.e., myocarditis) or inflammation of the membrane surrounding the heart (i.e., pericarditis) or both few months after recovery of COVID-19 patients $(12,13)$.

Based on the autopsy results, myocardial infection is observed in 35\% of deceased COVID-19 patients (14).

A high adipose tissue angiotensin converting enzyme (ACE2) expression in an obese person increases inflammatory responses. These responses lead to epicardial adipose tissue inflammation, and subsequently result in myocarditis (14).

Long-term cardiac complications of COVID-19 include arrhythmias, myocardial infarction (MI), heart failure, cardiogenic shock, exercise intolerance, and sudden cardiac death.

Cardiorenal syndrome (CRS) type 3 is also associated with some short-term and long-term cardiac complications. COVID-19-induced AKI can affect the heart in two ways;

1. Directly; through activating the renin-angiotensinaldosterone system (RAAS) and sympathetic nervous system

2. Indirectly; through volume overload, electrolyte disturbances, and acidemia (15).

\section{Blood vessel injury}

COVID-19 increases the risk of blood clot formation by increasing the risk of blood cell adhesions; hence, it can lead to heart attack and stroke, and can adversely affect the lungs, legs, liver, and kidneys (3).

This disease can also lead to weakness of blood vessels, acceleration of atherosclerosis, hypertension, formation of aortic aneurysms, and coronary artery disease (15).

\section{Nerve injury}

COVID-19-associated nerve injury depends on the infection course, treatment, and recovery (16).

SARS-COV-2 can enter the brain through blood and cerebrospinal fluid, and in rare cases through infecting the airways (17).

In the short term, COVID-19 causes brain injuries such as brain parenchymal inflammation, agitation, and confusion; however, its long-term effects include cognitive, sensory and behavioral disorders, smell and taste dysfunctions, stroke, epilepsy, Guillain-Barré syndrome, and neurodegenerative diseases (e.g. Parkinson's and Alzheimer's diseases).

In COVID-19 patients, a specific biological process leads to the loss of gray matter in a process similar to the formation of amyloid plaques in Alzheimer's disease, and thereby accelerates the development of Alzheimer's in these patients.

COVID-19 can also play a major role in the development of Alzheimer's disease by making substantial changes in the blood-brain barrier permeability.

Neutrophils can increase the risk of Alzheimer's disease through inflammations caused by new molecules (16). 
Mood problems and chronic fatigue syndrome (CFS)

ICU (intensive care unit) hospitalization certainly increases the risk of developing post-traumatic stress disorder, depression, anxiety, and sleep disorders $(2,3)$. Some long-term complications of COVID-19 are predicted with regard to the long-term complications of similar viruses such as SARS. CFS, which is caused by hyperinflammation and cytokine storm, is among these longterm complications. CFS (also known as extreme fatigue) gets worse with physical and mental activities, but it does not get better with rest $(3,17)$.

Symptoms of CFS include:

- Severe physical fatigue

- Brain fog

- Difficulty sleeping

- Muscle pain

- Fatigue after activity (6).

\section{Musculoskeletal injury}

Following their recovery, many ICU patients experience symptoms such as muscle fatigue, decreased ability to walk, loss of balance, mobility problems, decreased muscle strength, decreased muscle volume, changes in muscle fibers, and dysfunction of muscle contraction.

These complications occur due to:

1. ICU hospitalization, bed rest, and inactivity

2. The use of medications such as corticosteroids, and complications associated with ICU hospitalization (e.g., mechanical ventilation) which can lead to insulin resistance and myopathy (4).

In addition, in rare cases, the risk of developing viral infections such as hepatitis $\mathrm{B}$, infectious mononucleosis, and measles increases after recovery (7).

One year after the onset of COVID-19 pandemic, many long-term complications of the disease are still unknown; therefore, international scientific collaboration is required to identify these complications in the near future.

Authors' contribution

Both authors contributed equally to this manuscript.

\section{Conflicts of interest}

The authors declare that they have no competing interests.

Ethical considerations

Ethical issues (including plagiarism, data fabrication, double publication) have been completely observed by the authors.

\section{Funding/Support}

None.

\section{References}

1. Al-Jahdhami I, Al-Naamani K, Al-Mawali A. The Post- acute COVID-19 Syndrome (Long COVID). Oman Med J. 2021;36:e220. doi: 10.5001/omj.2021.91.

2. Harikrishnan P. Gustatory Dysfunction as an Early Symptom in COVID-19 Screening. J Craniofac Surg. 2020;31:e656-e658. doi: 10.1097/SCS.0000000000006797.

3. Olingy CE, Dinh HQ, HedrickCC. Monocyte heterogeneity and functions in cancer. J Leukoc Biol. 2019;106:309-322. doi: 10.1002/JLB.4RI0818-311R.

4. Sagarra-Romero L, Viñas-Barros A. COVID-19: short and longterm effects of hospitalization on muscular weakness in the elderly. Int J Environ Res Public Health. 2020;17:8715. doi: 10.3390/ijerph17238715.

5. Sisó-Almirall A, Brito-Zerón P, Conangla Ferrín L, Kostov B, Moragas Moreno A, Mestres J, et al. Long Covid-19: proposed primary care clinical guidelines for diagnosis and disease management. Int J Environ Res Public Health. 2021;18:4350. doi: 10.3390/ijerph18084350.

6. What are the long-term effects of COVID-19?. Available from: https://www.medicalnewstoday.com/articles/long-termeffects-of-coronavirus.

7. Yelin D, Wirtheim E, Vetter P, Kalil AC, Bruchfeld J, Runold M, et al. Long-term consequences of COVID-19: research needs. Lancet Infect Dis. 2020;20:1115-7. doi: 10.1016/S14733099(20)30701-5.

8. Greenhalgh $T$, Knight $M, A^{\prime}$ Court $C$, Buxton $M$, Husain L. Management of post-acute covid-19 in primary care. BMJ. 2020;370:m3026. doi: 10.1136/bmj.m3026.

9. George PM, Barratt SL, Condliffe R, Desai SR, Devaraj A, Forrest I, et al. Respiratory follow-up of patients with COVID-19 pneumonia. Thorax. 2020;75:1009-1016. doi: 10.1136/thoraxjnl-2020-215314.

10. Salehi S, Reddy S, Gholamrezanezhad A. Long-term pulmonary consequences of coronavirus disease 2019 (COVID-19): what we know and what to expect. J Thorac Imaging. 2020;35:W879. doi: 10.1097/RTI.0000000000000534.

11. Tao SL, Wang XM, Feng YG, Kang PM, Li QY, Sun TY, et al. Is the presence of lung injury in COVID-19 an independent risk factor for secondary lung cancer? Med Hypotheses. 2020;143:110074. doi: 10.1016/j.mehy.2020.110074.

12. Post-COVID Conditions. Available from: https://www.cdc.gov/ coronavirus/2019-ncov/long-term-effects.html.

13. Kawakami R, Sakamoto A, Kawai K, Gianatti A, Pellegrini D, Nasr A, et al. Pathological Evidence for SARS-CoV-2 as a Cause of Myocarditis: JACC Review Topic of the Week. J Am Coll Cardiol. 2021;77:314-325. doi: 10.1016/j.jacc.2020.11.031.

14. Mitrani RD, Dabas N, Goldberger JJ. COVID-19 cardiac injury: Implications for long-term surveillance and outcomes in survivors. Heart Rhythm. 2020;17:1984-90. doi: 10.1016/j. hrthm.2020.06.026.

15. Becker RC. Anticipating the long-term cardiovascular effects of COVID-19. J Thromb Thrombolysis. 2020;50:512-24. doi: 10.1007/s11239-020-02266-6.

16. Lennon JC. Neurologic and Immunologic Complications of COVID-19: Potential Long-Term Risk Factors for Alzheimer's Disease. J Alzheimers Dis Rep. 2020;4:217-21. doi: 10.3233/ ADR-200190.

17. Anushka J. Long Term Complications Associated with Covid-19: A Review. International Journal of Innovative Science and Research Technology. 2020;5:324-6. doi: 10.38124/IIISRT20SEP144. 